\title{
JMJ14 is an H3K4 demethylase regulating flowering time in Arabidopsis
}

Cell Research (2010) 20:387-390. doi: 10.1038/cr.2010.27; published online 23 February 2010

\section{Dear Editor,}

Histone lysine methylation plays an essential role in regulating chromatin functions such as transcription and heterochromatin formation. Histone H3 lysine 4 (H3K4) methylation is linked to active transcription $[1,2]$. Recent findings in mammals have demonstrated that histone methylation is reversible by a family of Jumonji C (JmjC) domain-containing proteins. KDM5/ JARID1 family proteins have been shown to be able to demethylate H3K4me1,2,3 in mammals [3]. Previously, we identified six proteins in Arabidopsis showing high sequence similarity to KDM5/JARID1 family proteins [4]. Here we demonstrate that one such protein, JMJ14, is an active histone $\mathrm{H} 3 \mathrm{~K} 4$ demethylase and is involved in flowering time regulation.

JMJ14 encoded by At4g20400 shows the highest sequence similarity to human KDM5/JARID1 family histone demethylases, suggesting that JMJ14 might be an active histone $\mathrm{H} 3 \mathrm{~K} 4$ demethylase. To test this prediction, we developed an in vivo assay to analyze its histone demethylase activity (Figure 1A). In brief, we first overexpressed JMJ14 in half of a 5-week-old tobacco leaf by injecting Agrobacteria carrying a FLAG-tagged $J M J 14$ driven by the $35 \mathrm{~S}$ promoter (Figure 1B). The other half of the leaf was not infiltrated and served as a control. Then nuclei were isolated from the leaf 2 days after infiltration and stained for specific histone modifications (Figure 1C). 4,6-Diamidino-2-phenylindole (DAPI) staining indicates the location of nuclei in each field. Anti-FLAG antibody was used to visualize the expression of the putative histone demethylase, thus showing which cells were successfully transfected. Histone demethylation activity was then monitored by comparing immunostaining between transfected and non-transfected cells. Using this method, we analyzed the demethylase activity of JMJ14. In FLAG-stained cells where JMJ14 was overexpressed, H3K4me3 and H3K4me 2 modifications were hardly detectable, and $\mathrm{H} 3 \mathrm{~K} 4 \mathrm{me} 1$ was much lower compared to cells without FLAG labeling (Figure $1 \mathrm{C})$. In addition, when one conserved iron-binding resi- due of JMJ14, His397, was mutated to Ala (H397A), the H3K4 demethylation activity was abolished (Supplementary information, Figure S1). In contrast, no differences of mono-, di- or tri-methylation levels at H3K9, H3K27 and H3K36 were observed between cells with or without FLAG labeling (Supplementary information, Figure S2). This demonstrates that JMJ14 is an H3K4-specific demethylase and the conserved iron-binding residue is absolutely required for its enzymatic activity.

The demethylation activity was also shown using an in vitro assay. JMJ14-YFP-HA and JMJ14H397A-YFP-HA proteins were transiently overexpressed in tobacco leaves and immunoaffinity purified using anti-HA antibody. Then, an in vitro demethylation assay was carried out using calf thymus histone as a substrate. The results were consistent with our in vivo assays that JMJ14 but not JMJ14H397A demethylated H3K4me3,2,1, while H3K9me3, H3K27me3 and H3K36me3 were not affected (Figure 1D). Taken together, these results suggest that JMJ14 has the enzymatic activity and specificity to reverse $\mathrm{H} 3 \mathrm{~K} 4 \mathrm{me} 3$, H3K4me2 and $\mathrm{H} 3 \mathrm{~K} 4 \mathrm{me} 1$. Moreover, JMJ14 was localized to cell nuclei in both tobacco leaves and transgenic Arabidopsis expressing the JMJ14-YFPHA fusion protein (Supplementary information, Figure S3), further supporting the conclusion that JMJ14 is a histone demethylase in vivo.

To investigate the roles of JMJ14 in plant development, we obtained two T-DNA insertion mutants, jmj14-1 and jmj14-2, from the SALK T-DNA collection. The JMJ14 gene (At4g20400) contains 12 exons and encodes a 954-amino-acid polypeptide with distinct domains, including the JmjN domain, JmjC domain, C5HC2 zinc finger (zf-C5HC2), F/Y-rich N-terminus (FYRN) and F/ Y-rich C-terminal (FYRC) domains [4]. The T-DNAs inserted into the sixth exon (jmj14-1) and the eighth intron (jmj14-2) of $J M J 14$, respectively (Figure 1E). $J M J 14$ full-length cDNA was not detectable in either of these two mutants by RT-PCR (Figure 1F). Compared to the wild-type plants, both of the mutants showed an early flowering phenotype under long day (LD, 16-h light and 8-h dark) conditions (Figure $1 \mathrm{G}$ and $1 \mathrm{H}$ ). 

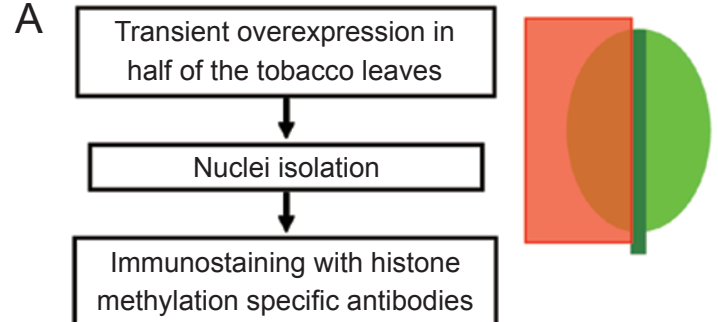

C
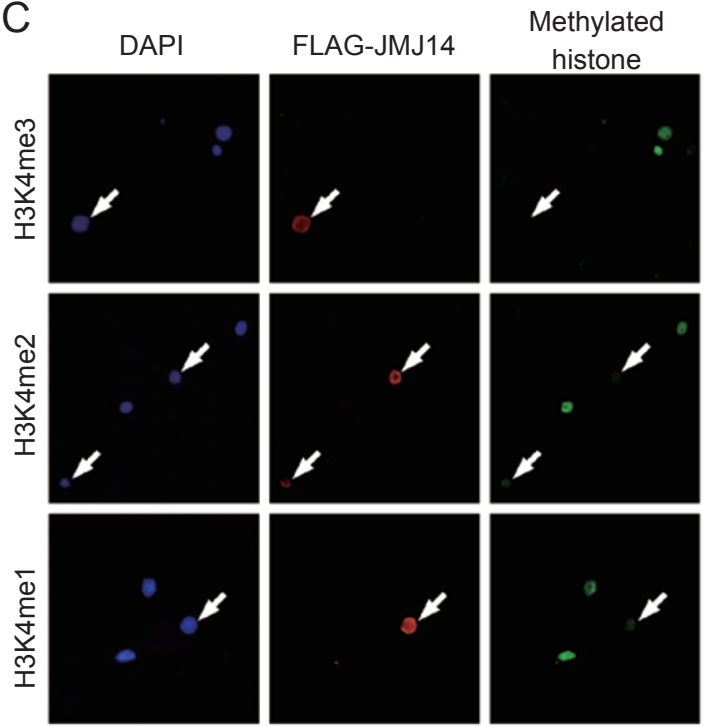

E

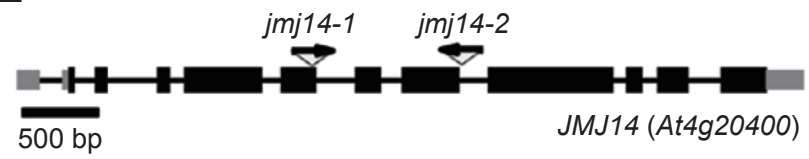

F
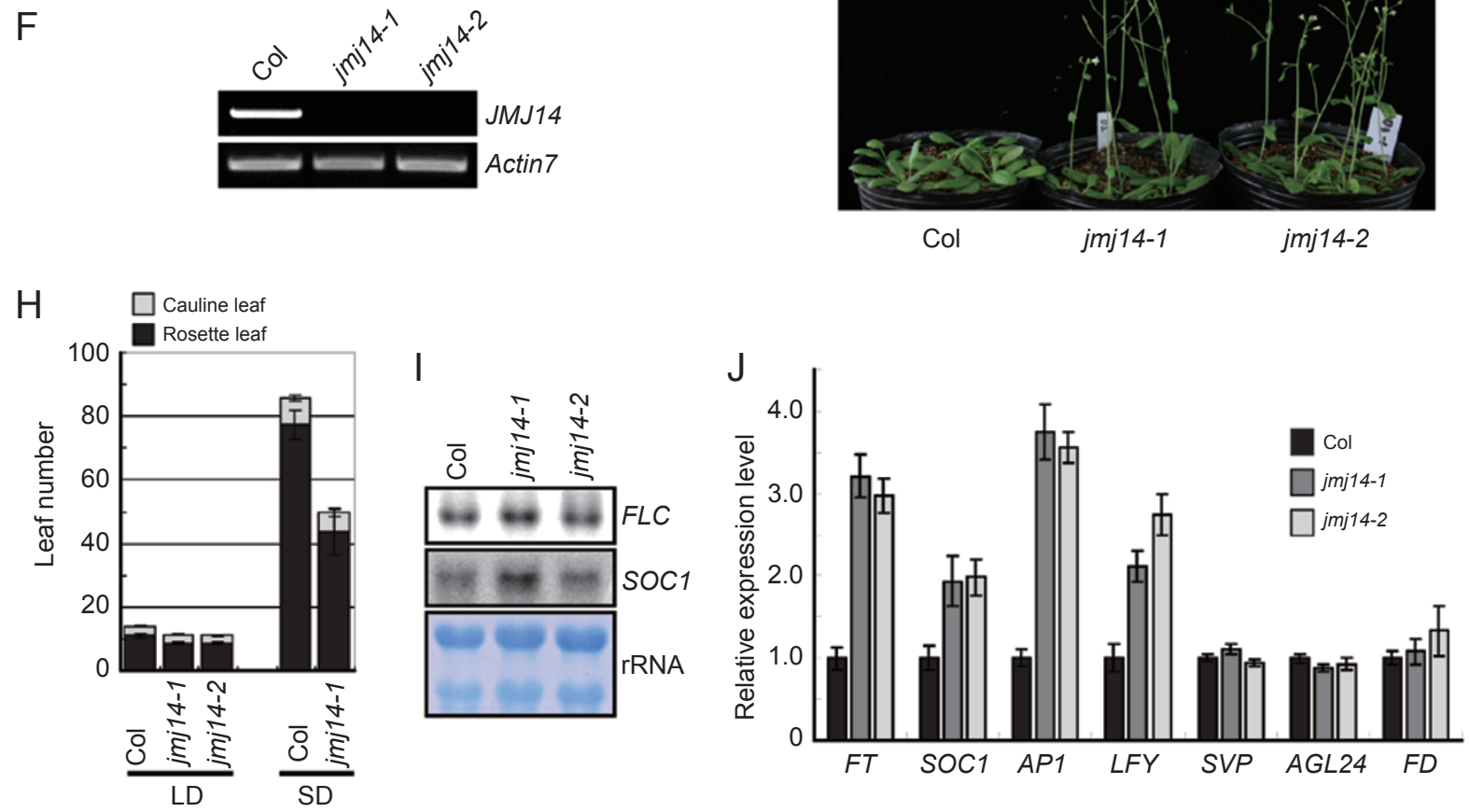
Floral transition is one of the most important developmental processes of higher plants and must be executed properly for maximum reproductive success. In Arabidopsis, flowering time is controlled by internal and environmental cues and can be affected by four major pathways, namely photoperiod, vernalization, gibberellic acid signaling, and autonomous pathways [5]. FLC is a major repressor of flowering. The Short Vegetative Phase (SVP) protein was shown to physically interact with FLC to repress flowering [6]. Floral signals are integrated by floral integrators FLOWERING LOCUS $T$ (FT), SUPPRESSOR OF OVEREXPRESSION 1 OF CONSTANS (SOC1), AGAMOUS-LIKE 24 (AGL24), APETALA1 (APl) and LEAFY $(L F Y)$. FD, a bZIP protein, interacts with FT in the shoot apex to promote SOC1 expression [7]. To explore the pathway in which JMJ14 acts, we further assayed the jmj14-1 phenotype in short day (SD) conditions. The jmj14-1 mutant was early flowering under SD conditions; however, it is much later than that in LD conditions (Figure $1 \mathrm{H}$ ), suggesting that jmj14 mutants respond normally to photoperiod. The response of jmj14-1 to different photoperiods is similar to that of $F L C$-dependent pathway mutants. Therefore, we investigated the $F L C$ transcript level in jmj14 mutants. The FLC expression level in jmj14-1 was similar to that in wild-type plants, indicating that $J M J 14$ regulation of flowering time acts independently from $F L C$ expression and it probably regulates downstream floral integrators (Figure 1I). To test this hypothesis, we examined the ex- pression levels of floral integrators that act cooperatively with $F L C$ or downstream of $F L C$, including SOC1, $L F Y$, $F T, A P 1, S V P, A G L 24$, and $F D$. RNA gel blot analysis showed a slight elevation of SOC1 transcripts (Figure 1I). Quantitative RT-PCR analysis showed that transcripts of FT, SOC1, $L F Y$ and $A P 1$ were 2- to 4-fold increased from wild-type levels in jmj14 mutants, whereas the expression of $S V P, A G L 24$ and FD remained unchanged (Figure 1J). Thus, the early flowering phenotype of jmj 14 mutants was associated with increased expression of floral integrators.

As $\mathrm{H} 3 \mathrm{~K} 4 \mathrm{me} 3$ is a hallmark of active transcribing regions and JMJ14 is a histone $\mathrm{H} 3 \mathrm{~K} 4$ demethylase, we wondered whether overexpression of $F T, A P 1$ and SOC1 was caused by increased H3K4 methylation due to loss of JMJ14. A chromatin immunoprecipitation (ChIP) assay was used to examine the H3K4 methylation status of FT, AP1 and SOC1 chromatin loci. Chromatin regions used in ChIP assays were as previously reported, except for the $F T$ promoter region (FT P) and SOC1 D (Supplementary information, Figure S4). ChIP results showed that $\mathrm{H} 3 \mathrm{~K} 4 \mathrm{me} 2$ and $\mathrm{H} 3 \mathrm{~K} 4 \mathrm{me} 3$ methylation levels were not significantly different between jmj 14 mutants and wild type at the $A P 1$ locus, the $F T$ locus or the SOC1 locus (Supplementary information, Figure S4). Therefore, JMJ14 repression of flowering time might not act through directly demethylating $F T, A P 1$ or $S O C 1$ loci.

The FT locus has been previously shown to possess

Figure $1 \mathrm{JMJ} 14$ is a histone H3K4 demethylase that represses flowering. (A) Scheme of the in vivo histone demethylation assay is shown on the left and a diagram of an infiltrated leaf is shown on the right. The red shadow indicates the part of the leaf infiltrated. (B) Diagrams of constructs used in FLAG-JMJ14 and JMJ14-YFP-HA transient expression. (C) Overexpression of FLAG-JMJ14 reduced H3K4me3, H3K4me2 and H3K4me1. Histone methylation was visualized by immunostaining with rabbit polyclonal modification specific antibodies followed by Alexa Fluor 488 conjugated goat anti-rabbit (green), which is shown in the right panels. Nuclei transfected with FLAG-JMJ14 were detected by immunostaining with FLAG M2 antibody followed by Alexa Fluor 555 conjugated goat anti-mouse (red) and are shown in the middle panels. Nuclei were revealed by DAPI staining (blue) and are shown in the left panels. More than 50 pairs of transfected nuclei versus non-transfected nuclei were observed with the same results. Arrows indicate nuclei transfected with FLAG-JMJ14. (D) JMJ14-YFP-HA but not JMJ14H397A-YFP-HA, which contains histidine 397 to alanine mutation in the Fe(II) binding site, can demethylate H3K4 methylation on calf thymus histone in vitro. However, JMJ14-YFP-HA has no effect on H3K9me3, H3K27me3 and H3K36me3. MBP (maltose binding protein) was used as a no enzyme control. The methylation status was detected by immunoblots with methylation-specific antibodies. Immunoblot with histone $\mathrm{H} 3$ showed equal loading. (E) Gene structure of the JMJ14 transcribed unit and T-DNA insertion site of jmj14 mutants. Black bars, gray bars and black lines indicate coding exons, UTRs and introns, respectively. T-DNA insertions are indicated by triangles, directions of which are indicated by arrows. Bar equals $500 \mathrm{bp}$. (F) RT-PCR of full-length JMJ14 revealed that transcription of JMJ14 was eliminated in jmj14 mutants. Actin7 was used as an internal control. (G) jmj14 mutants show early flowering under LD (long day, 16-h light, 8-h dark) conditions. (H) jmj14 mutants were early flowering under both LD and SD (short day, 8-h light, 16-h dark) conditions. Black cylinders represent rosette leaf number and gray cylinders represent cauline leaf number. Error bars represent $2 \times$ standard error. (I) RNA gel blot analysis of FLC (upper) and SOC1 (middle) in 10 DAG seedlings grown under LD. Membrane post transfer stained with methylene blue revealing rRNA was used as a loading control (bottom). (J) Quantitative RT-PCR of FT, SOC1, AP1, LFY, SVP, AGL24, and FD using 10 DAG seedlings grown under LD. Transcript levels were first normalized to Actin7 expression levels in each sample, and then normalized to the expression of the Col sample. Black bars represent Col, dark gray bars represent jmj14-1 and light gray bars represent jmj14-2. Error bars represent 2× standard error. 
H3K4me3, which could be repressed by H3K27me3 catalyzed by CURLY LEAF (CLF) [8]. In clf mutants, H3K4me3 was increased 2- to 4-fold at the FT locus. However, we note that $c l f$ mutants had about a 200 fold increase in $F T$ transcription [8]. In this work, we showed that jmj14 mutants had about a 3-fold increase in $F T$ transcription, which is unlikely to be caused by a significant change in $\mathrm{H} 3 \mathrm{~K} 4$ tri-methylation. Recently, one related study reported that $\mathrm{H} 3 \mathrm{~K} 4 \mathrm{me} 3$ was increased at the FT transcription initiation region and that JMJ14 (called JMJ4 in their study) associated with the FT promoter directly [9]. One possibility of this difference is that materials of different developmental stage were used in the experiments. Jeong et al. used 57-day-old SD grown plants, whereas we used 10-day-old LD grown plants. In addition, they showed that a 200-bp I region of FT promoter was enriched four times by JMJ14-FLAG; in contrast, an overlapping 200-bp G region showed no enrichment in our assays.

Although the JmjC domain is essential for histone demethylase activity, such activity may not always be associated with the function of cognate proteins. For example, the function of Lid2, a yeast homolog of KDM5/ JARID1, in euchromatin does not require its demethylation activity but requires an intact JmjC domain [10]. As for JMJ14's function in repressing flowering, the requirement of its demethylation activity remains to be tested in further study. If repressing $F T, S O C 1, A P 1$ and $L F Y$ expression by JMJ14 requires its demethylation activity, we would also expect that some histone H3K4 methyltransferases should be involved in promoting their expression.

In conclusion, our study has revealed that JMJ14 is a histone H3K4 demethylase that is important for preventing early flowering through repression of the floral integrators $F T, A P 1, S O C 1$ and $L F Y$ during the vegetative growth phase.

(Experimental materials and methods are depicted in the Supplementary information, Data S1)

\section{Acknowledgments}

We thank Dr T Jenuwein for providing us the H3K27me3 antibody and Qingbao Zhu for technical support. We thank the Arabidopsis Biological Resources Center at Ohio State for pro- viding SALK T-DNA-insertion lines. This work was supported by grants from the National Basic Research Program of China (2009CB941500 and 2005CB522400 to XC) and the National Natural Science Foundation of China (30771209 to CL, and 30930048 and 30921061 to $\mathrm{XC}$ ).

Falong $\mathrm{Lu}^{1,2, *}$, Xia Cui ${ }^{1, *}$, Shuaibin $\mathrm{Zhang}^{1,2}$, Chunyan Liu ${ }^{1}$, Xiaofeng Cao ${ }^{1}$

${ }^{I}$ National Key Laboratory of Plant Genomics and Center for Plant Gene Research, Institute of Genetics and Developmental Biology, Chinese Academy of Sciences, No. 1, Bei Chen West Road, Chaoyang District, Beijing 100101, China; ${ }^{2}$ Graduate School, Chinese Academy of Sciences, Beijing 100049, China

*These two authors contributed equally to this work

Correspondence: Xiaofeng Cao

Tel: +86-10-64869203; Fax: +86-10-64873428

E-mail: xfcao@genetics.ac.cn

\section{References}

1 Jenuwein T, Allis CD. Translating the histone code. Science 2001; 293:1074-1080.

2 Kouzarides T. Chromatin modifications and their function. Cell 2007; 128:693-705.

3 Klose RJ, Zhang Y. Regulation of histone methylation by demethylimination and demethylation. Nat Rev Mol Cell Biol 2007; 8:307-318.

4 Lu F, Li G, Cui X, Liu C, Wang XJ, Cao X. Comparative analysis of JmjC domain-containing proteins reveals the potential histone demethylases in Arabidopsis and rice. J Integr Plant Biol 2008; 50:886-896.

5 Putterill J, Laurie R, Macknight R. It's time to flower: the genetic control of flowering time. Bioessays 2004; 26:363-373.

6 Li D, Liu C, Shen L, et al. A repressor complex governs the integration of flowering signals in Arabidopsis. Dev Cell 2008; 15:110-120.

7 Michaels SD. Flowering time regulation produces much fruit. Curr Opin Plant Biol 2009; 12:75-80.

8 Jiang D, Wang Y, He Y. Repression of FLOWERING LOCUS $\mathrm{C}$ and FLOWERING LOCUS T by the Arabidopsis Polycomb repressive complex 2 components. PLoS ONE 2008; 3:e3404.

9 Jeong J-H, Song H-R, Ko J-H, et al. Repression of FLOWERING LOCUS T chromatin by functionally redundant histone H3 lysine 4 demethylases in Arabidopsis. PLOS ONE 2009; 4:e8033.

10 Li F, Huarte M, Zaratiegui M, et al. Lid2 is required for coordinating $\mathrm{H} 3 \mathrm{~K} 4$ and $\mathrm{H} 3 \mathrm{~K} 9$ methylation of heterochromatin and euchromatin. Cell 2008; 135:272-283.

(Supplementary information is linked to the online version of the paper on the Cell Research website.) 